\title{
Dynamic Performance of an Excitation System Built in a Digital Way
}

\author{
M.L. Orozco, H. Vásquez \\ ${ }^{1}$ Universidad del Valle, Escuela de Ingenier a El ctrica y Electr nica, \\ Cali, Colombia, \\ email:maloroz@hotmail.com \\ ${ }^{2}$ Universidad del Valle, Escuela de Ingenier a El ctrica y Electr nica, Cali, Colombia, email: vasquez@univalle.edu.co
}

\begin{abstract}
This paper presents the results obtained in the performance of a digital excitation system designed with digital signal processor (DSP). The modeling of the plant, the structure of the synchronous generator terminal voltage control and the models of limiters were developed as routines or programmed algorithms in the DSP.
\end{abstract}

\section{INTRODUCTION}

An excitation system performs control and protective functions for a synchronous generator. In generation plants exist a great variety of this equipment, which have been constructed with different technologies and using different control structures. Initially these systems were developed with analog electronic, after hybrid systems were built (including analog and digital circuits) and finally on the last decade excitation systems were implemented in digital way, making good use of the advantages of speed, computing power, flexibility and remote access of these devices [1]. Figure 1 shows the functional block diagram of a typical excitation control system for a synchronous generator.

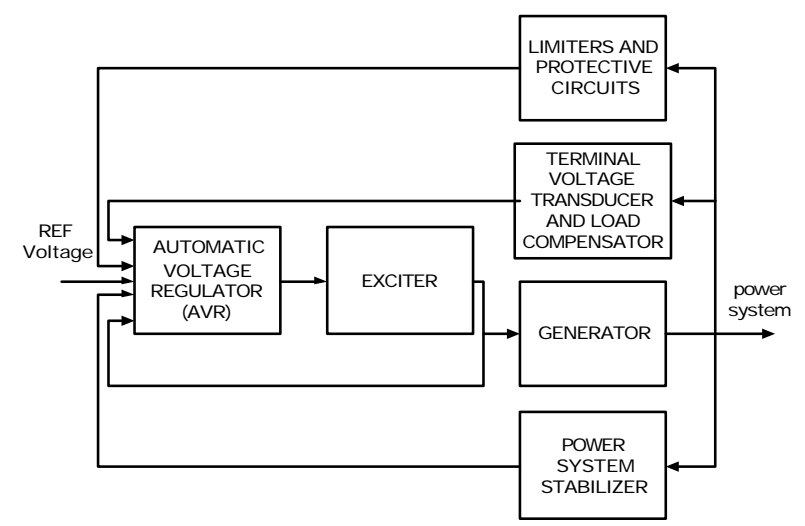

Figure 1. Functional block diagram of a synchronous generator excitation control system [2]

The main element of the excitation system is the automatic voltage regulator. This maintains the machine terminal voltage in a desired reference, which can be varied in order to accomplish different power system requirements. The excitation system includes limiting and protective functions, to ensure that the capability limits of the exciter

and synchronous generator are not exceeded. Some of these functions are overexcitation limiter, underexcitation limiter, overcurrent limiter and volts/Hertz limiter. On the other hand the power system stabilizer provides an additional input signal to the regulator to damp power system oscillations. The exciter provides dc power to the synchronous machine field winding; it could be another de machine or a static converter. The transducers convert the voltage or currents levels to adequate values to be process by the control unit [2].

The purpose of this paper is to present a digital excitation system based on a digital signal processor (DSP). The structure of the synchronous generator terminal voltage control and some limiters models were developed in digital way, as programmed algorithms in the DSP.

\section{GENERAL SYSTEM STRUCTURE}

The digital excitation system was developed over a digital signal processor; the DSP starts the capture of variables, performs mathematical operations and control algorithms and sends the control signals to the actuator. Automatic voltage regulator (automatic/manual control), overexcitation limiter, underexcitation limiter and volts/Hertz limiter are included in this digital excitation system The exciter is a totally controlled single-phase SCR's bridge. The excitation system is from selfexcited type, therefore the exciter is fed with the generated voltage.

\section{III.AUTOMATIC AND MANUAL VOLTAGE CONTROL}

The main control system is the automatic voltage regulator (AVR); it is implemented with a PI strategy that guarantees zero stationary state error and a good response speed. Also a field voltage control (manual control), was developed. The control strategy applied in this case is only proportional action, due to the field loop is only a gain, because the DSP linearizes the actuator.

\section{A. Linearization of Actuator}

The actuator is a rectifying totally controlled single-phase bridge, whose transfer function is: 


$$
V c d=\frac{2 V m}{\pi} \cos \alpha
$$

where:

$$
\begin{aligned}
& \mathrm{Vm}=\text { Peak of Terminal Voltage } \\
& \alpha=\text { Fire angle }
\end{aligned}
$$

The cosine function, of the nonlinear type, can be linearized using previously an arcosine function; in this case it was not necessary to use an external board, because the DSP has a library with trigonometric functions stored.

Figure 2 illustrates the linearized block diagram from the controller output to the exciter input .

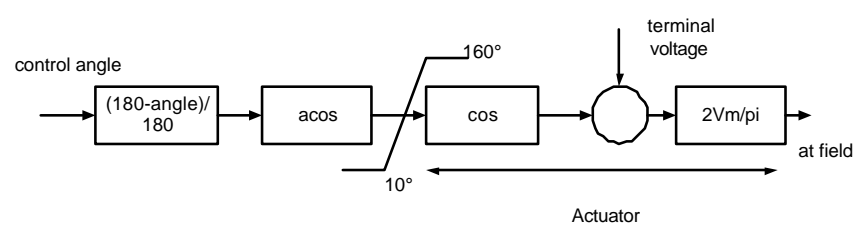

Figure 2. Block diagram of linear actuator

Finally, is just a direct loop gain, from the way out of the control to the generator's field.

\section{B. Control Loop}

Figure 3 shows the block diagram for the terminal voltage and field voltage regulation. Normally the system operates on automatic mode, therefore the regulation comes true on the terminal voltage; however, circunstances exist, i.e. a possible fault in the terminal voltage measurement, so the system has to operate in manual mode; in this case the controlled variable is the field voltage. The operation of manual control excludes the PI action and only works with a proportional action. Limiters also are excluded in this operation, because they modify the AVR's terminal voltage reference.

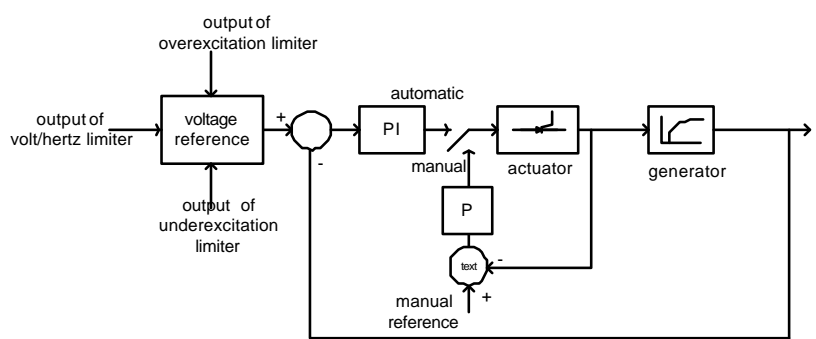

Figure 3. Block Diagram of Automatic Control Voltage and Manual Control

The change from automatic mode to manual mode, or viceversa, must to success without transient oscillations; in order to guarantee this operation, a follower function that maintains the firing angle in the same value, at the moment of the change is developed.

\section{No load Answers}

Figures 4 and 5 show the results obtained with the implementation of all functions in digital way; obtained data were kept in the DSP as historic data. The DSP was used as a central processing unit. The system was sampled every 16,67 $\mathrm{ms}$. The operation of the digital control for the automatic and manual mode shows the following results:

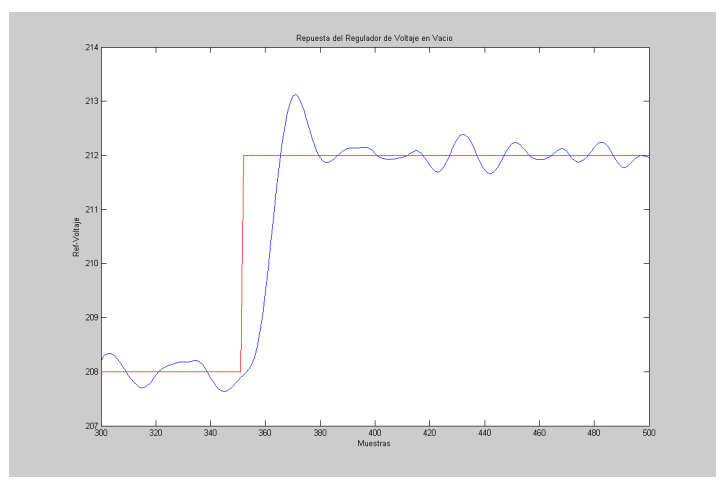

Figure 4. Response of Automatic Voltage Control

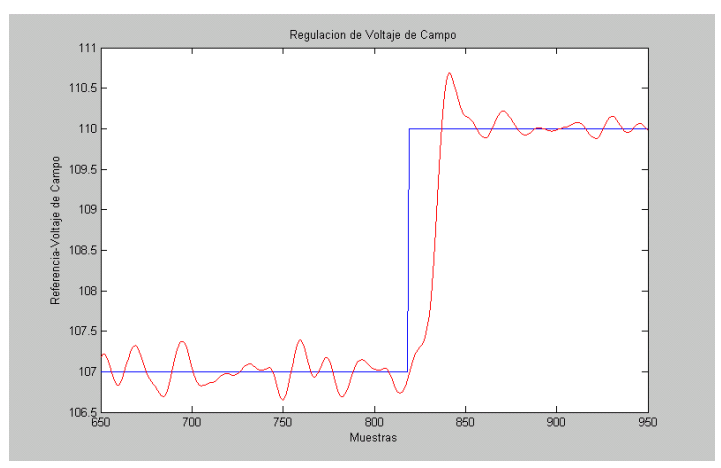

Figure 5. Response of Manual Control

As it is observed in the figure 4, the answer of the terminal voltage due to a $5 \%$ reference change, presents an overshoot of $25 \%$ and a zero stationary state error. For control in manual mode (figure 5), is observed the field voltage dynamic due to a $5 \%$ reference change; the answer shows a zro stationary state error and a $20 \%$ overshoot. The settling time remains between $600 \mathrm{~ms}$ and $800 \mathrm{~ms}$.

When the generator is connected to the power system, the voltage is imposed by the system conditions due to it behaves like an infinite bus. Therefore, when the generator is connected to the power system, the deliver of reactive power to the system is regulated. In this case, the measurement of terminal voltage is complemented with a reactive current component, to built the terminal voltage transducer.

The value of the crosscurrent component is given by:

$\mathrm{I}_{\text {sin }}$ component $=\beta * I_{\text {line }} * \operatorname{sen} \varphi$ 
Where: $\beta=$ weight of $I_{\text {sin }}$

$\mathrm{I}_{\text {line }}=$ Line Current

$\varphi=$ angle between terminal voltage and line current

Generally in the early excitation systems this function was implemented with external hardware; with the utilization of the DSP as a brain of arithmetic operations, we used trigonometric functions that are available at its mathematical library; for this reason the implementation of this function was very simple.

The obtained results with the generator connected to the power grid are observed in the figure 6 :

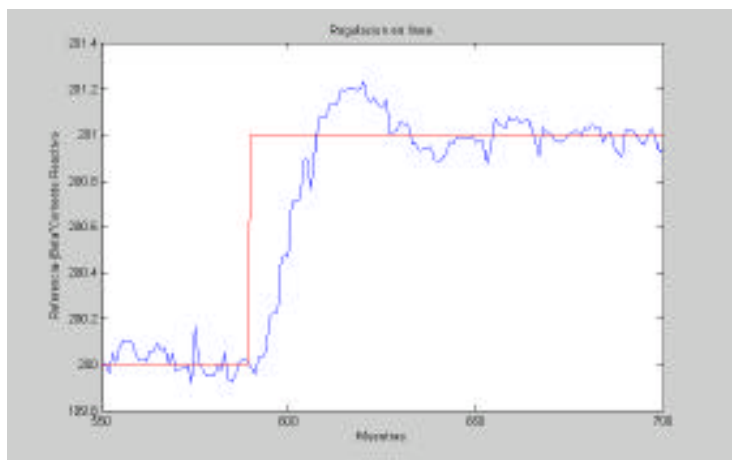

Figure 6. Response of Automatic Voltage Regulator from a machine connected to the power grid

\section{LIMITERS}

Limiters are functions executed when the generator enters in a status of dangerous operation, which can cause damages as overheating, overfluxing and loss of synchronism. The limiters take the operating point to a state inside of the limits of the machine's capability. [2], [4]. Limiters can be: overexcitation limiter, underexcitation limiter, overvoltage limiter, overcurrent limiter, voltage/frequency $(\mathrm{V} / \mathrm{Hz})$ limiter, although they can include some others, for example: the power factor limiter and reactive power limiter, among others. For this application the overexcitation limiter, underexcitation limiter and $\mathrm{V} / \mathrm{Hz}$ limiter were developed in the DSP.

\section{A. Overexcitation Limiter(OEL)}

The overexcitation limiter prevents an overheating in the machine's field winding. The limiting functions can ake control of the system, in this case deactivating the regulator operation or providing an additional input signal to the voltage regulator error summing junction; therefore the voltage regulator continues in operation but its reference is alterate by the limiter's output signal. Under normal conditions when the OEL is not limiting, $\mathrm{V}_{\mathrm{OEL}}$ would be normally zero; when the maximum value of excitation is excedeed the limiter is activated and generates a negative signal which is added to the voltage regulator reference, then it a decrease of the total reference is produced; the dynamic effect is that the controller used for the terminal voltage control loop will follow the total reference; therefore the excitation ramps move the limiter setpoint from the instantenous value to the timed limiter setting. The way to reach this desired point has different configurations: some limiters operate with a inverse time characteristic, other generate a ramp as a function of the overexcitation quantity [2], [4], [5].

The model utilized for the overexcitation limiter is observed in the figure 7.

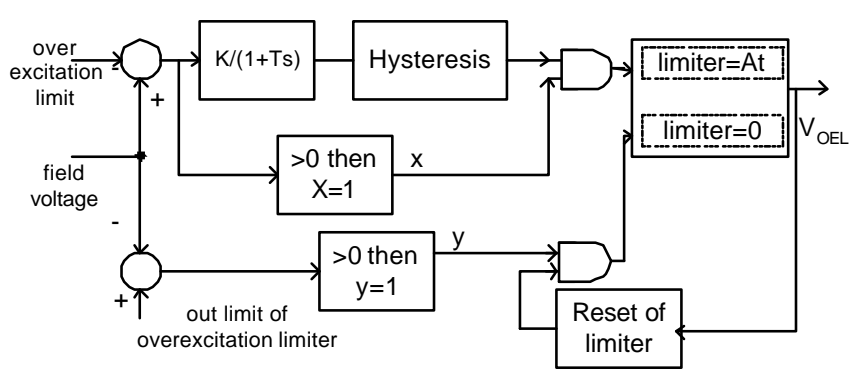

Figure 7. Model of Overexcitation limiter

A first order dynamics is observed, it is get into in order to secure that the limiter enters to work when overexcitation is not a low transient; besides it takes care that for the high previous voltage to overexcitation, the performance of the limiter will be faster compared to previous lower levels. The first order output is taken to a comparator with hysteresis that permits deactivate the limiter when the field voltage is lower to the limiter setpoint. This dead band is a voltage range where the limiter will get stable, the output is retained but it is not cancelled. The characteristic of this dead band permits insuring that the limiter is deactivated totally when the field voltage is less than the maximum allowed level. If the limiter don't have the hysteresis band, it would generates a decrease of the field voltage, but this level would be vary close to get into overexcitation again. This is possibly when the limiter returns to zero.

The responses of the overexcitation limiter are shown in the figures 8 and 9 .

In this case the maximum overexcitation limit is $135 \mathrm{~V}$; when the field voltage reaches this maximum point it presents temporary delay to initiate its descent to a safe operation point. This delay corresponds to a first order dynamics in the model.

The figure 9 shows the evolution of voltage reference, it decreases until the generator leaves the overexcitation. 


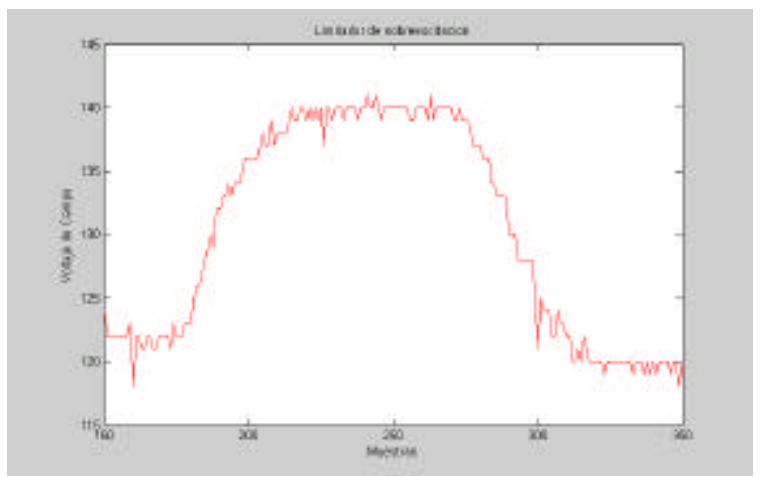

Figure 8. Field Voltage

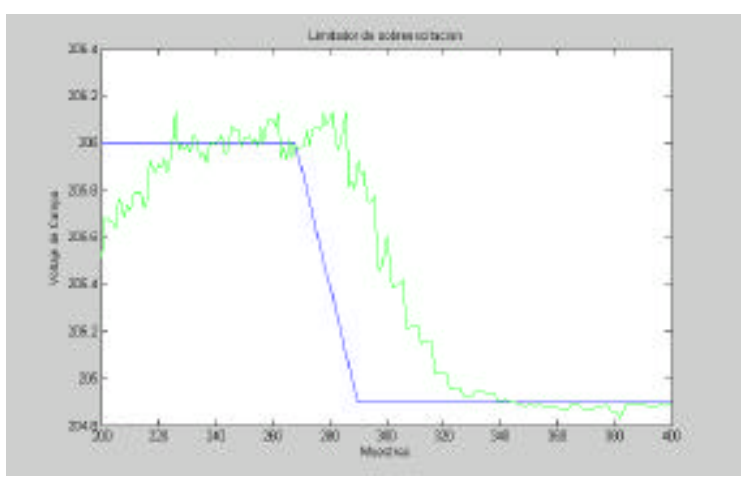

Figure 9. Voltage Reference (blue) and Terminal Voltage (green)

\section{B. Underexcitation Limiter (UEL)}

The underexcitation limiter avoids the machine loss of synchronism or avoids an overheating in the stator end region, due to his close operation to the minimal limit of the machine's capability curve [2],[4]. The underexcitation limiters can perform in two ways: Taking control of the system through a diode in a High Value (HV) gate. In this case the UEL takes over control, replacing the normal voltage regulator signal; the limiter needs a considerable gain, in that way the AVR should be replaced. In other applications the UEL is given control by providing an additional input signal to the voltage regulator error summing point [6],[7].

The IEEE presents three underexcitation limiters models [6], where the input to this models are the voltage and current or active and reactive power, as well as the maximum limit that varies in terms of the terminal voltage. The figure 10 shows one of the possible characteristics of the underexcitation limiter [6]; as it is observed, the traced straight line represents the maximum limit permitted for relations of Active Power Vs. Reactive Power, which can be determined by the machine's capability curve .

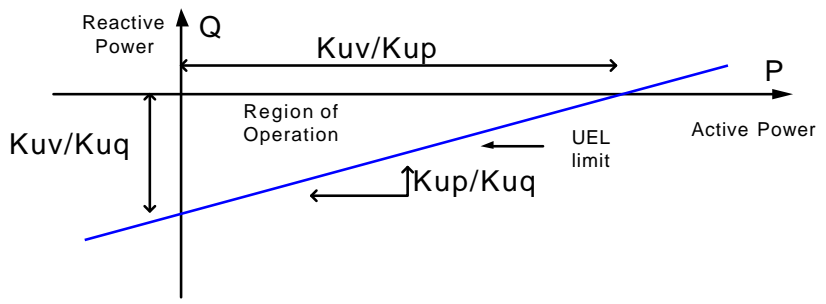

Figure 10. Straight line limiting characteristic of an underexcitation limiter [6]

The used model is observed in figure 11:

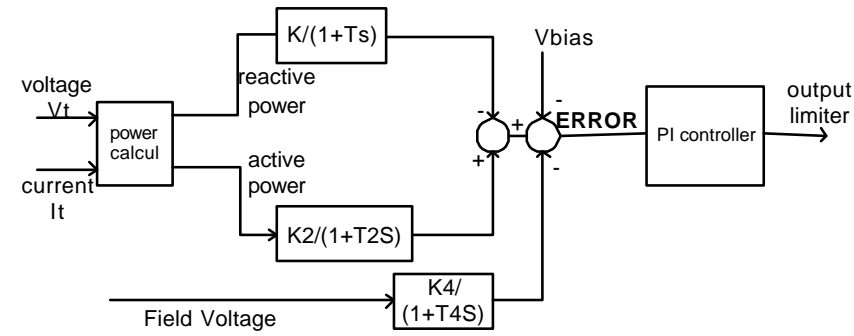

Figure 11. Simplified Model of Underexcitation limiter [6]

The limiter acts when the error signal is positive, that initiates the limiter action (PI) and produces a signal that is added to the AVR's reference; the effect over the voltage control is an excitation raising.

The advantage to develop the limiter in this way is that it has a measure of how far is the machine operating point in relation to the limiting characteristic; for example for far values of the limit line, the error signal generated is high; in the moment when the excitation is returning to the safe operation zone, the error signal is reduced and the limiter effect is less. The disadvantage is that the limiter forces the machine to operate on the limit line, where the error signal becomes zero, causing a status of underexcitation again.

Obtained results are the following:

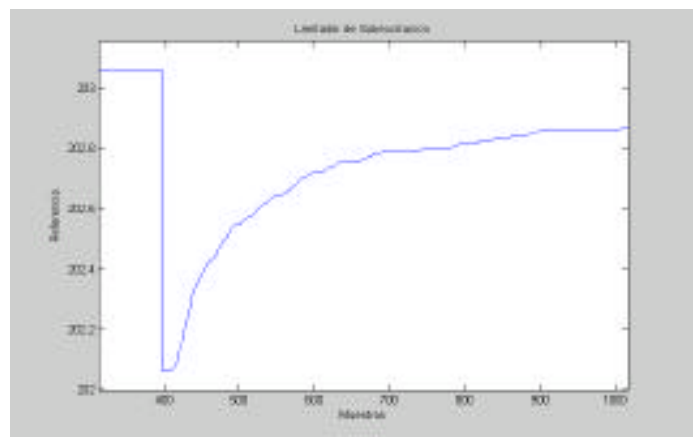

Figure 12. Response of Underexcitation limiter (Voltage reference) 


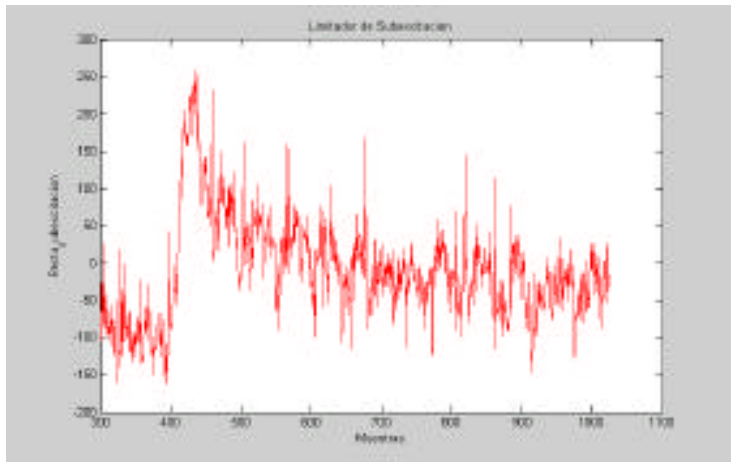

Figure 13. Response of Underexcitation limiter (error signal)

Figure 12 shows the regulator voltage reference. Initially applies a change of reference in order to carry the generator to the underexcitation status; when is detected the limiter is started in soft form and its output is added to the voltage reference; insofar as the system leaves the underexcitation state, the reference returns to one stable operation point. The dynamics is slow due to the low values for the constants.

In the figure 13 error value is observed; the overshoot is taken around zero due to the limiter action in a slow way, when the generator enters to underexcitation state.

\section{Voltage/Frecuency Limiter}

The Volt/Hertz limiter receives the terminal voltage and its frequency as input signals, and protects the synchronous machine and the transformers connected to it damages by conditions of overflux [2],[4],[5].

The overflux is due to a big relation between voltage and frequency, caused for a suddenly frequency drop.

Typical limits to bring about the Volt/Hertz limiter are 1.05 pu or $1.1 \mathrm{pu}$. This limiter can operate in join whit he overvoltage limiter (limiter that prevents an excess of terminal voltage)[5].

The model used is observed in the figure 14 [5]. The model inputs are the terminal voltage and the frequency. It has an additional input to the stabilizer of the excitation system. The input signal filter is not used for this application, because its effect in not important in comparison with the dynamics of the system, and the frecuency measure is done using the terminal voltage measure.

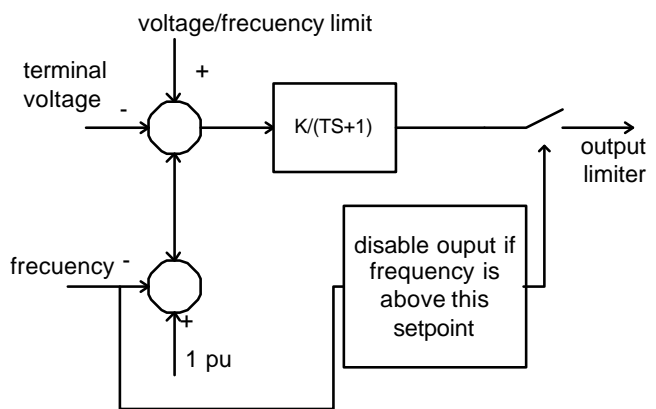

Figure 14. Simplified Model of the V/Hz Limiter
Answers obtained with the $\mathrm{V} / \mathrm{Hz}$ limiter are shown in the figures 15,16 and 17 .

In this case the used limit is $1,05 \mathrm{pu}$, which is presented when the frequency has decreased lower than 57 hertz (figure 15); when the relation $\mathrm{V} / \mathrm{Hz}$ is bigger than $1,05 \mathrm{pu}$, the limiter output is added to the voltage reference. The model used reduces the terminal voltage in the same proportion in that the frequency drops (figure 16). The evolution of the relation voltage/frequency is observed in the figure 17, showing that this relation presents small increments insofar as the frequency drops and the voltage increases; in this case also is observed a little overshoot due to the transient answer of the terminal voltage.

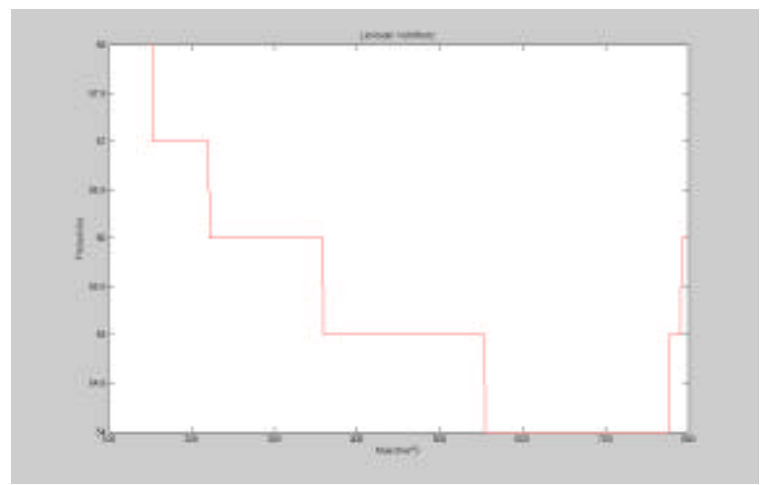

Figure 15. Response of V/Hz Limiter (frecuency)

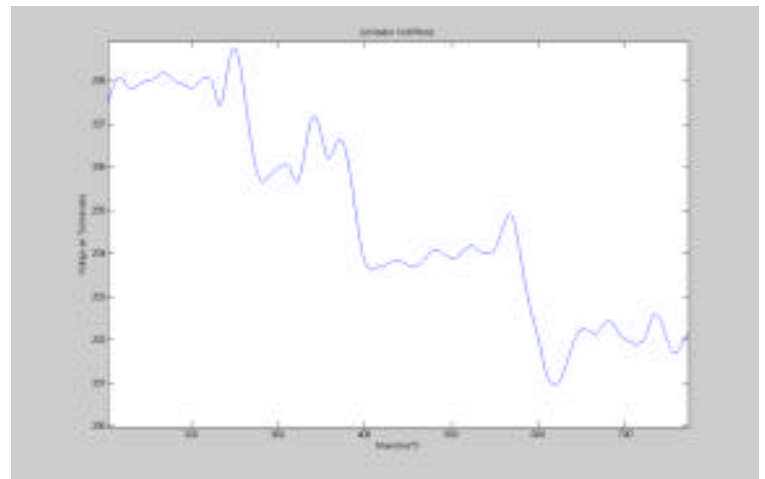

Figure 16. Response of V/Hz Limiter (Terminal Voltage)

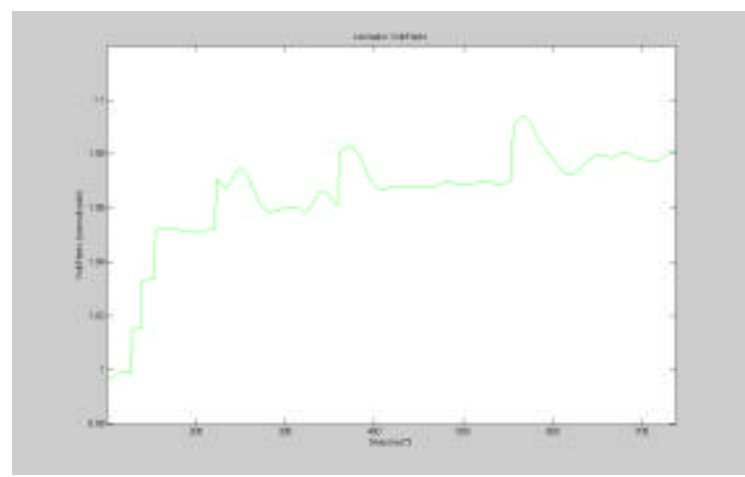

Figure 17. Response of the $\mathrm{V} / \mathrm{Hz}$ Limiter 


\section{CONCLUSIONS}

The digital excitation system was developed using a DSP as a central process unit; every mathematical and control functions and the routines of command and protection were developed on the DPS. The structure utilized for the task execution allowed a good performance of the system dynamics. Manual and automatic control functions using proportional action and proportional-integral action were enough for obtaining fast response speed and a zero state stationary error. The overexcitation limiter implemented using a first order dynamics permits that the limiter operates faster when previous overexcitation levels are high; on the other hand the hysteresis zone avoids oscillations around the limit point when the limiter is given back to zero. The underexcitation limiter presents a good performance, however oscillations around the operation limit zone were observed, because its structure moved the generator operation point to the limit line. The operation of the $\mathrm{V} / \mathrm{Hz}$ limiter was adequated; when is presented a relation between voltage/frecuency on top of 1.05 $\mathrm{pu}$ decreased the voltage in the same proportion that the frecuency drops, avoiding an excessive overflux. Future implementations require to reject the oscillations presented on the underexcitation limiter, and the overshoots in the voltage /frequency limiter.

\section{REFERENCES}

[1] Digital Excitation Applications Task Force, Digital Excitation Technology - A Review Of Features, Functions and Benefits, IEEE Transactions on Energy Conversion, Vol. 12, No.3, 1997.

[2] P. Kundur, Power System Stability and Control, Ed. Mc Graw Hill, 1993.

[3] M. L Orozco, Dise o, Construcci n e Implementaci $n$ de un Sistema de Excitaci n Digital para Un Generador Sincr nico Interconectado a la Red El ctrica, Universidad del Valle, 2005.

[4] George K. Girgis, Hoa D. Vu, Verification Of Limiter Performance in Modern Excitation Control Systems, IEEE Transactions on Energy Conversion, Vol. 10, No.3, September 1995 Pag. 538

[5] IEEE Task Force on Excitation Limiters, Recommended Models for Overexcitation Limiting Devices, IEEE Transactions on Energy Conversion, Vol. 10, No.4, December 1995, Pag 706.

[6] IEEE Task Force on Exciation Limiters, Underexcitation Limiter Models For Power System Stability Studies, IEEE Transactions on Energy Conversion, Vol. 10, No.3, September 1995, Pag 524
[7] G. Roger, Les M., R.E. Beaulieu, An Utility perspective on Under-Excitation Limiters, IEEE Transactions on Energy Conversion, vol. 10, No. 3, 1995.

M. L. Orozco. Electronic Engineer . M.Sc in Automatic of Universidad del Valle in Colombia, where she has worked in control system, power electronics and voltage regulation.

H. Vasquez. Electrical Engineer of Universidad del Valle in Colombia. Diplom Ingenieur der Elektrotechnik, Technische Universität Braunschweig, Alemania. He is working in Universidad del Valle in area of Automatic. He comes at present director from the School of Electrical and Electronic Engineering of Universidad del Valle (Colombia). 